\title{
PERÍODOS E IMAGENS DAS FUNÇÕES SENO E COSSENO: Uma verificação por meio do software Geogebra
}

\author{
Alan Silva dos Santos \\ Marcelo Eduardo Pereira \\ Marco Antônio Di Pinto ${ }^{(*)}$
}

\section{INTRODUÇÃO}

O ensino das funções trigonométricas seno e cosseno tem sido objeto de investigações nacionais e internacionais. No âmbito nacional, temos alguns exemplos de dissertações de mestrado envolvendo esta temática: Costa (1997) realizou uma sequência didática envolvendo o uso do computador para investigar a aprendizagem de trigonometria e obteve conclusões que apontam para uma contribuição positiva ao aluno com relação ao entendimento e a construção do conhecimento matemático. Oliveira (2010) investigou uma abordagem para o ensino de trigonometria desde os conceitos envolvidos no triângulo retângulo até o ciclo trigonométrico e funções trigonométricas, construiu uma sequência de aplicativos que usam o software GeoGebra, fundamentando o ciclo e funções trigonométricas além da utilização das tecnologias não digitais (transferidor, régua e compasso). Rosenbaum (2010) faz uso de resultados de pesquisas para o trabalho com funções trigonométricas por meio de atividades que envolvem resolução de problemas por meio de construções de tecnologias não digitais como régua e compasso e do uso de tecnologias digitais como calculadora científica e o software GeoGebra. Concluiu-se que o uso de pesquisas contribuiu para a organização do ensino de funções trigonométricas. Ribeiro (2011) investiga os conhecimentos prévios dos alunos de funções trigonométricas e trigonometria em utilizar o caderno do aluno da rede pública do estado de São Paulo, e aponta que a dificuldade encontrada pelos alunos está relacionada aos conteúdos de procedimentos e de atitudes. Porém, ao utilizar as tecnologias não digitais como lápis, papel, transferidor, régua e compasso houve a construção do conhecimento matemático pelos alunos. Tavares (2013) realizou um estudo das relações entre os coeficientes e representações gráficas entre as funções seno e cosseno por meio do software

\footnotetext{
${ }^{(*}$ Alan Silva dos . Mestre em Educação Matemática pela Pontifícia Universidade Católica de São Paulo. E-mail: alan.objetivo@gmail.com.
}

Marcelo Eduardo Pereira. Mestre em Educação Matemática pela Pontifícia Universidade Católica de São Paulo. Atualmente é professor da Universidade Metropolitana (Unimes). E-mail: marcelo.pereira@unimes.br.

Marco Antônio Di Pinto. Mestre em Educação Matemática pela Pontifícia Universidade Católica de São Paulo. Atualmente é professor da Universidade Metropolitana (Unimes). E-mail: marco.antonio@unimes.br. 
Winplot, propondo um novo estudo em relação às funções trigonométricas. Os autores fizeram abordagens em suas pesquisas envolvendo o uso das tecnologias não digitais e tecnologias digitais para o ensino e a aprendizagem de funções trigonométricas.

As constatações providas por meio das investigações já mencionadas por estes pesquisadores inspiraram uma proposta em relação a este trabalho que procura empregar uma visão com a utilização das tecnologias digitais na educação matemática, tendo como público alvo alunos do curso de licenciatura em matemática.

Uma sequência didática foi elaborada envolvendo a utilização do software GeoGebra. Este estudo envolve uma aplicação com tecnologias digitais (modelo digital) e tecnologias não digitais (lápis e papel), para verificação de períodos e imagens das funções seno e cosseno, as simulações realizadas no software são um recurso de mediação junto com a sequência didática para com os alunos.

Neste artigo está descrito o uso do applet, que tem por base as representações dos períodos e imagens das funções seno e cosseno, na forma geométrica, que buscam favorecer um ambiente de construção e assimilação do conhecimento matemático, os alunos poderão fazer uso dos controles deslizantes, utilizar comandos como caixa de entrada do próprio software, além de utilizar o zoom para mais verificações quanto as oscilações das funções.

\section{OBJETO MATEMÁTICO}

Os objetos matemáticos utilizados nesta pesquisa são os períodos e imagens das funções seno e cosseno, os autores a seguir definem as funções seno e cosseno como:

As funções cos: $\mathbb{R} \rightarrow \mathbb{R}$ e sen: $\mathbb{R} \rightarrow \mathbb{R}$, chamadas função cosseno e função seno respectivamente, são definidas pondo-se, para cada $t \in \mathbb{R}$ :

$E(t)=(\cos t, \operatorname{sen} t)$

Noutras palavras, $\mathrm{x}=\cos t$ e $\mathrm{y}=\operatorname{sen} t$ são respectivamente a abcissa e a ordenada do ponto $\mathrm{E}(\mathrm{t})$ da circunferência unitária.

Segue-se imediatamente desta definição que vale, para todo $t \in \mathbb{R}$, a relação fundamental

$\cos ^{2} t+\operatorname{sen}^{2} t=1$

Uma função $f: \mathbb{R} \rightarrow \mathbb{R}$ chama-se periódica quando existe um número $T \neq 0$ tal que $f(t+T)=f(t)$ para todo $t \in \mathbb{R}$. Se isto ocorre, então $f(t+T)=f(t)$ para todo $t \in \mathbb{R}$ e todo $k \in \mathbb{Z}$. O menor número $\mathrm{T}>0$ tal que $f(t+T)=f(t)$ para todo $t \in \mathbb{R}$ chama-se o período da função $f$. As funções seno e cosseno são periódicas, de período $2 \pi$. (LIMA et al., 2012, p.257)

Os autores referem-se a $\mathrm{x}=\cos t$ como abcissa e $\mathrm{y}=\operatorname{sen} t$ é a ordenada do ponto $\mathrm{E}(\mathrm{t}) \mathrm{da}$ circunferência unitária. Escrevem que as funções cosseno e seno são periódicas, com período $2 \pi$. 
Os cálculos dos períodos e imagens das funções seno e cosseno podem ser realizados por meio das tecnologias não digitais (lápis e papel) e sua verificação pode ser obtida por meio das tecnologias digitais (modelo digital), onde mais adiante sua explicação estará explícita.

$$
\begin{array}{ll}
\text { i) } f(x)=a+b \cdot \operatorname{sen}(c x+d) & P=\frac{2 \pi}{\| d \mid} \\
\text { ii) } f(x)=a+b \cdot \cos (c x+d) & P=\frac{2 \pi}{\| d \mid}
\end{array}
$$

A seguir é possível fazer a verificação quanto aos períodos e imagens das funções seno e cosseno. Em relação à função $f(x)=2 \operatorname{sen}\left(\frac{x}{2}\right)$ tem-se:

P = $\frac{p}{\| a \mid}=\frac{2 \pi}{\left|\frac{1}{2}\right|}=4 \pi \quad ; \operatorname{Im}=[-2 ; 2]$
\begin{tabular}{|c|c|c|}
\hline$\frac{x}{2}$ & $\operatorname{sen}\left(\frac{x}{2}\right)$ & $2 \operatorname{sen}\left(\frac{x}{2}\right)$ \\
\hline 0 & 0 & 0 \\
\hline$\frac{\pi}{2}$ & 1 & 2 \\
\hline$\pi$ & 0 & 0 \\
\hline$\frac{3 \pi}{2}$ & -1 & -2 \\
\hline $2 \pi$ & 0 & 0 \\
\hline
\end{tabular}

Em relação à função $g(x)=3 \cos \left(\frac{x}{2}\right)$ tem-se:

$P=\frac{p}{|a|}=\frac{2 \pi}{\left|\frac{1}{2}\right|}=4 \pi \quad ; \operatorname{Im}=[-3 ; 3]$

\begin{tabular}{|c|c|c|}
\hline$\frac{x}{2}$ & $\cos \left(\frac{x}{2}\right)$ & $3 \cos \left(\frac{x}{2}\right)$ \\
\hline 0 & 1 & 3 \\
\hline$\frac{\pi}{2}$ & 0 & 0 \\
\hline$\pi$ & -1 & -3 \\
\hline$\frac{3 \pi}{2}$ & 0 & 0 \\
\hline $2 \pi$ & 1 & 3 \\
\hline
\end{tabular}

Por meio das tecnologias não digitais tem-se a verificação quanto ao período e imagem das funções seno e cosseno. Assim como por meio das tecnologias digitais que será visitada mais adiante. Para isto os pressupostos metodológicos que compõem esta pesquisa vêm a seguir. 


\title{
FUNDAMENTAÇÃO E METODOLOGIA
}

Fundamenta-se este trabalho na teoria das situações didáticas de Guy Brousseau, com o intuito de modelar o processo de ensino e de aprendizagem no que se refere aos conceitos matemáticos.

\begin{abstract}
Uma "situação" é um modelo de interação de um sujeito com um meio determinado. O recurso de que esse sujeito dispõe para alcançar ou conservar um estado favorável nesse meio é um leque de decisões que dependem do emprego de um conhecimento preciso. Consideramos o meio como subsistema autônomo, antagônico ao sujeito. Assim, ao tomarmos como objeto de estudo as circunstâncias que regem a difusão e a aquisição dos conhecimentos, vamos nos interessar pelas situações. (BROUSSEAU, 2008, p.21).
\end{abstract}

A teoria das situações didáticas é estabelecida por momentos sendo estes momentos: adidática quando envolve (o saber e o aluno), didática quando envolve (o professor, saber e o aluno), de devolução (caracteriza-se quando o aluno toma para si a responsabilidade de resolver o problema), de institucionalização (identifica-se um novo saber construído pelos alunos, em que há a intervenção do professor).

Se em determinada situação o professor entrega o problema ao aluno e este por sua vez recorre ao professor para que ele lhe ofereça subsídios para solução. Neste momento gera-se um milieu sendo este um fator de desequilíbrio que envolve o aluno e o saber, onde o aluno busca estratégias para solucionar o problema proposto. Nesse momento tem-se a devolução, pois o aluno toma para si a responsabilidade, buscando estratégias para solução do problema proposto.

A teoria das situações didáticas envolve as seguintes dialéticas: ação (o aluno está para agir frente à situação proposta), formulação (momento de troca de informações do aluno com uma ou várias pessoas), validação (o aluno através do modelo por ele criado mostra validade) e institucionalização (é fixado o estatuto cognitivo do saber pelo professor).

Articulando neste enfoque a teoria das situações didáticas a esta pesquisa, no primeiro momento de ação, no quadro 1 mais adiante, mostra a atividade a ser proposta ao sujeito, o aluno precisará buscar conceitos, informações com base em seus conhecimentos prévios e começar a agir frente a situação proposta, neste momento o sujeito está a selecionar os conceitos a serem utilizados para possível solução das questões.

No segundo momento de formulação, o sujeito busca formular as possíveis soluções, começando a conjecturar. Neste momento está a testar seus conceitos, e buscar soluções para resolver a questão, dispõe-se de estratégias, podendo começar, errar e retornar, e buscar uma solução ótima para tal questão. 
No terceiro momento o sujeito busca validar suas hipóteses, seus conceitos, que utilizou a fim de resolver a questão, após os momentos de ação e formulação, ele busca validar suas hipóteses por meio de verificações, neste momento de validação o aluno poderá utilizar o quadro 2 , isto é, a utilização do software GeoGebra, fazer uso da movimentação do controle deslizante, e em seguida observar e confrontar os resultados obtidos por meio de suas soluções no quadro 1.

O quarto momento é o de institucionalização, onde o professor/educador, fixa o estatuto cognitivo do saber, é o momento que um novo saber será fixado com o saber antigo do educando, o aluno poderá reformular seu pensamento quanto à solução da questão, ou ainda, poderá acrescentar uma nova solução que ainda não possuía em seus saber antigo. A seguir têm-se as descrições no que se refere às interfaces computacionais.

Como recurso de verificação incluso na teoria das situações didáticas, o aluno deve mostrar validade do modelo por ele criado pela dialética da validação. Esta validação pode ser realizada por meio das tecnologias não digitais (lápis e papel) e tecnologias digitais (envolve modelo digital). Assim tem-se a seguinte descrição pelo autor:

Um modelo digital não é lido ou interpretado como um texto clássico, ele geralmente é explorado de forma interativa. Contrariamente a maioria das descrições funcionais sobre papel ou dos modelos reduzidos analógicos, o modelo informático é essencialmente plástico, dinâmico, dotado de uma certa autonomia de ação e reação. Como Jean-Louis Weissberg observou tão bem, o termo simulação conota hoje esta dimensão interativa, tanto quanto a imitação ou a farsa. (LÉVY, 1993, p.121)

Lévy (1993) escreve em sua obra que um modelo digital não é lido ou interpretado do modo de um texto clássico, mas é explorado geralmente de forma interativa. Um modelo digital gera um conhecimento por simulação, sobre o conhecimento por simulação tem-se a seguinte observação do autor:

O conhecimento por simulação, menos absoluto que o conhecimento teórico, mais operatório, mais ligado as circunstâncias particulares de seu uso, junta-se assim ao ritmo sociotécnico específico das redes informatizadas: o tempo real. A simulação por computador permite que uma pessoa explore modelos mais complexos e em maior número do que se estivesse reduzido aos recursos de sua imagística mental e de sua memória de curto prazo, mesmo se reforçadas por este auxiliar por demais estático que é o papel. (LÉVY, 1993, p.125-126).

O modelo digital pode ser chamado de applet, que é um software que executa uma atividade específica dentro de outro software, este por sua vez depende do programa Java para ser executado.

O conhecimento por simulação permite que o aluno explore modelos mais complexos e em maior número, o dinamismo exposto nos softwares por meio do modelo digital (applets), 
proporciona ao aluno um conhecimento em curto prazo, uma vez que as simulações no software permitem a execução de comandos com rapidez. A investigação aqui descrita é de caráter qualitativo, e envolve como sujeitos, alunos do curso de licenciatura em matemática, a participação do estudo ocorre de forma voluntária. A aplicação da sequência didática ocorrerá em duas sessões, composta por dois blocos de duas horas cada.

O material utilizado nesta pesquisa é composto pela sequência didática exposta no quadro 1 e pelo modelo digital (applet) na figura 1. Este modelo digital permite a verificação da imagem e período das funções seno e cosseno por meio da execução de comandos no software GeoGebra. O aluno ao movimentar o controle deslizante formará graficamente os períodos das funções seno e cosseno e a imagem.

Figura 1: Modelo Digital (applet) para verificação de período e imagem das funções seno e cosseno.

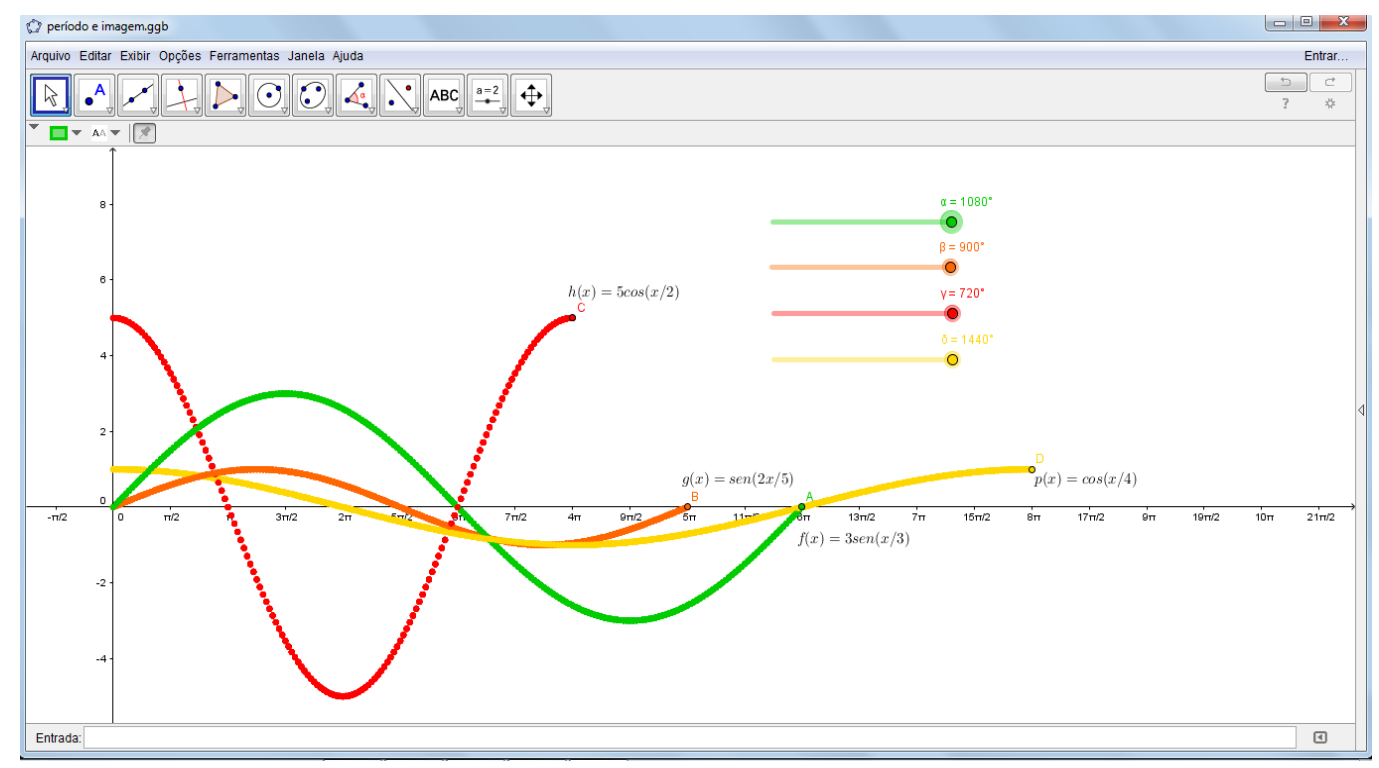

Fonte: Dos autores.

As atividades no quadro 1 foram utilizadas a fim de mediar este estudo. Na atividade 1 solicita-se para que o aluno encontre as soluções dos períodos e imagens das funções com o uso das tecnologias não digitais (lápis e papel), com base em seus conhecimentos prévios sobre trigonometria e funções trigonométricas. Na atividade 2 solicita-se para que os alunos façam a verificação quanto as imagens e períodos das funções trigonométricas utilizando as tecnologias digitais (modelo digital) e transcrevam os valores obtidos após a verificação. 
Quadro 1. Estudo dirigido utilizado com os sujeitos da pesquisa
Atividade 1
Resolva o período e imagem das funções a seguir:
a) $f(x)=3 \operatorname{sen}\left(\frac{x}{6}\right)$
b) $g(x)=\operatorname{sen}\left(\frac{2 x}{5}\right)$
c) $h(x)=5 \cos \left(\frac{x}{2}\right)$
d) $p(x)=\cos \left(\frac{x}{4}\right)$

Atividade 2

Por meio do software GeoGebra faça a verificação quanto aos períodos e imagens das funções seno e cosseno e transcreva os valores obtidos.
a) $f(x)=3 \operatorname{sen}\left(\frac{x}{6}\right)$
b) $g(x)=\operatorname{sen}\left(\frac{2 x}{5}\right)$
c) $h(x)=5 \cos \left(\frac{x}{2}\right)$
d) $p(x)=\cos \left(\frac{x}{4}\right)$

Fonte: os autores

A análise das respostas dos alunos poderá permitir reflexões quanto ao conceito de períodos e imagens das funções seno e cosseno pelos alunos.

\section{CONSIDERAÇÕES FINAIS}

Quanto à finalização deste artigo, os alunos já estavam selecionados, e as sessões agendadas na instituição. Esta é uma proposta que aborda a utilização do software GeoGebra a fim de favorecer a construção do conhecimento matemático. Uma vez observada as análises pode-se prosseguir com este estudo e observar quais as concepções dos sujeitos quanto a utilização do software GeoGebra para a representação de período e imagem das funções seno e cosseno. Este artigo ainda permite a ampliação desta aplicação para outros tipos de funções que poderão ser abordadas para alunos do curso de licenciatura em matemática. 


\section{REFERÊNCIAS}

BROUSSEAU, G. Introdução ao estudo das situações didáticas: conteúdos e métodos de ensino. São Paulo: Ática. 2008.

COSTA, N. M. L. Funções seno e cosseno: uma sequência de ensino a partir de contextos do "mundo experimental" e do computador. 1997. Dissertação (Mestrado em Educação Matemática), PUC/SP.

LÉVY, P. As Tecnologias da Inteligência: o futuro do pensamento na era da informática, Rio de Janeiro: Editora 34. 1993.

LIMA, E. L; et al. A matemática do ensino médio. Volume 1. 10 ed. SBM. Rio de Janeiro. 2012. (Coleção professor de matemática)

OLIVEIRA, T. Trigonometria: A mudança da prática docente mediante novos conhecimentos. 2010. Dissertação de Mestrado, Universidade Federal de São Carlos, UFSCAR- São Paulo.

ROSENBAUM, L.S. Uma trajetória hipotética de aprendizagem sobre funções trigonométricas numa perspectiva construtivista. 2010. Dissertação (Mestrado em Ensino de Matemática). - Faculdade de Educação, Pontifícia Universidade Católica de São Paulo, 2010.

RIBEIRO, M.R.R.C. Possibilidades e dificuldades no desenvolvimento de situações de aprendizagem envolvendo funções trigonométricas. 2011. Dissertação (Mestrado Educação Matemática) -. Pontifícia Universidade Católica de São Paulo, São Paulo.

TAVARES, W.S. $O$ ensino das funções trigonométricas com o auxílio do software matemático de ambiente gráfico winplot. 2013. Dissertação (Mestrado em Educação) - Faculdade de Educação, Universidade Federal de Goiás.

\section{RESUMO}

Este estudo tem por objetivo propor uma abordagem de verificação de períodos e imagens das funções seno e cosseno por meio do software GeoGebra. Uma sequência didática foi elaborada tendo como proposta as tecnologias digitais (modelo digital) e as tecnologias não digitais (lápis e papel). Este estudo está apoiado em ideias da Teoria das Situações Didáticas. Trata-se de uma pesquisa que envolve uma proposta de ensino, com as representações gráficas por meio do software GeoGebra, realizando as simulações para verificação de períodos e imagens das funções trigonométricas, tendo como público alvo alunos do curso de licenciatura em matemática.

Palavras-chave: período; imagem; teoria das situações didáticas; GeoGebra.

\section{PERIODS AND IMAGES OF FUNCTIONS SINE AND COSINE AN VERIFICATION THROUGH SOFTWARE GEOGEBRA \\ ABSTRACT}

This study aims to propose a verification approach periods and images of sine and cosine functions through software GeoGebra. A teaching sequence was drawn up as proposed digital technologies (digital model) and non-digital technologies (pencil and paper). This study is supported by ideas of the Theory of Didactic Situations. It is a search that involves a teaching proposal with the graphical representations through the GeoGebra software, performing simulations for verification periods and images of the trigonometric functions, having as target students in mathematics degree course.

Keywords: period; image; theory of didactic situations; GeoGebra. 